\title{
Validación Preliminar de la Escala de Ansiedad ante la Muerte de Templer en una Muestra Argentina
}

\section{Preliminary Validation of Templer's Death Anxiety Scale in an Argentinean Sample}

\author{
Santiago Resett, Maia Kenserbaum y Pablo González Caino \\ Instituto de Ciencias Sociales y Disciplinas Proyectuales, Universidad Argentina de la Empresa
}

\begin{abstract}
La Escala de Ansiedad ante la Muerte de Templer es uno de los instrumentos mundialmente más usados a este respecto. Sin embargo, dicha escala no se ha adaptado en Sudamérica. De este modo, el presente trabajo examinó sus propiedades psicométricas en una muestra argentina. Se constituyó una muestra intencional de 859 adultos emergentes de Ciudad Autónoma de Buenos Aires y Gran Buenos Aires, Argentina (30\% varones, con edades de 18 a 30 años). Los participantes contestaron la Escala de Ansiedad ante la Muerte de Templer, las Escalas Ansiedad y Depresión de la Escala DASS-21 de Lovibond y Lovibond, el Inventario de Preocupación de Pensilvania de Meyer y otros y un cuestionario demográfico. Mediante análisis factoriales exploratorios y confirmatorios se comprobó una estructura bifactorial que era más adecuada que una estructura unifactorial de la escala. La consistencia interna, confiabilidad compuesta y confiabilidad test/retest fueron satisfactorias. Con respecto a su validez concurrente, se observaron correlaciones entre la escala y las medidas de ansiedad, depresión y preocupación — $r$ s de tamaño pequeño y mediano que iban de 0,28 a 0,48- Estos resultados indicarían que esta versión preliminar de la escala mostró evidencias de validez y confiabilidad en la Argentina. En la discusión se analizan las implicancias de estos hallazgos y se brindan sugerencias para futuros estudios, como estudiar sus propiedades en otras naciones de Sudamérica y evaluar su validez concurrente con otras medidas de ansiedad ante la muerte.
\end{abstract}

Palabras clave: ansiedad, muerte, Templer, validación, Argentina

Templer's Death Anxiety Scale is one of the most widely used instruments for evaluating death-related anxiety. However, this scale has never been adapted in South America. Thus, the present study examined the psychometric properties of this scale in an Argentinean sample. An intentional sample was constituted, comprising 859 emerging adults from Buenos Aires, Argentina (30\% male, aged 18 to 30 years). All participants completed Templer's Death Anxiety Scale, the Anxiety and Depression Scales of Lovibond and Lovibond's DASS-21 Scale, Meyer et al.'s Penn State Worry Questionnaire, and a demographic questionnaire. Exploratory and confirmatory factor analyses were carried out, resulting in a two-factor structure that proved to be more appropriate than the scale's one-factor structure. Also, internal consistency, composite reliability, and test/retest consistency were found to be satisfactory. Regarding concurrent validity, correlations were observed between the scale and measures of anxiety, depression, and worry-small and medium sized $r$ s ranging from 0.28 to $0.48-$. These results suggest that this preliminary version of the scale showed evidence of validity and reliability in Argentina. In the discussion, the implications of these findings are analysed and suggestions for future research are made; for instance, studying the scale's properties in other South American countries and evaluating its concurrent validity with other measures of death anxiety.

Keywords: anxiety, death, Templer, validation, Argentina

No temas a la muerte, y no temerás a la vida.

Epicuro

\section{Conceptualización de la Ansiedad ante la Muerte}

La muerte es un hecho natural, universal e irreversible que constituye — por dichas características—una de las fuentes de impacto psicológico más significativas para una persona (Lehto \& Stein, 2009; Neimeyer, 2015; Tong et al., 2016). Por lo tanto, la muerte no es un hecho instantáneo, sino que es un proceso biológico,

Santiago Resett iD http://orcid.org/0000-0001-7337-0617

El presente trabajo se basa parcialmente en la Tesis de Grado de la Universidad Argentina de la Empresa de la segunda autora.

La correspondencia relativa a este artículo debe ser dirigida a Santiago Resett, Instituto de Ciencias Sociales y Disciplinas Proyectuales, Universidad Argentina de la Empresa, Laurencena 222 bis, Paraná, Entre Ríos, Argentina. Email: santiago_resett@hotmail.com 
psicosocial, espiritual y cultural (Grau Abalo et al., 2008), generando intensas emociones en las personas, como ira o tristeza.

Una de las emociones más fuertes que puede generar la muerte es la ansiedad (Tomás-Sábado \& GómezBenito (2003). Limonero García (1997) y Limonero García et al. (2010) plantean que la ansiedad ante la muerte se puede definir como una reacción emocional que se produce a partir de la percepción de una amenaza a la propia existencia - en base a una situación real o imaginaria - que se desencadena a consecuencia de estímulos ambientales, como ver un cadáver o el fallecimiento de un familiar, o internos, como pensamientos o evocación de imágenes relacionadas con la muerte (Limonero García, 1997; Tomás-Sábado \& Gómez-Benito, 2004). Por otra parte, estas amenazas pueden ser iniciadas por estímulos que pongan en riesgo nuestra propia existencia o la de los demás —un amigo o un familiar-.

A pesar de que la muerte es un hecho humano universal, según Limonero García et al. (2010), la forma en que las personas experimentan la ansiedad ante la muerte y su manera de expresarlo varía en cada caso. Algunas de las variables que pueden introducir diferencias a este respecto son las sociodemográficas (la edad, el sexo, la religión, entre otras), por un lado, y las psicológicas, como la autoeficacia o autoestima, la personalidad o los estilos de afrontamientos, por el otro (Limonero García et al., 2010; Tomás-Sábado \& Gómez-Benito, 2003). Sin embargo, Sevilla-Casado y Ferré-Grau (2013) señalan que hay dos variables fundamentales que influyen en el grado de ansiedad ante la muerte de las personas: la salud mental y las experiencias cercanas a la muerte que una persona haya experimentado (accidentes o enfermedades terminales). No obstante, Tomás-Sábado y Gómez-Benito (2003) postulan que la ansiedad ante la muerte no la experimentan únicamente las personas que se encuentran enfermas físicamente, sino que son emociones que todos pueden experimentar al momento de pensar en la muerte. Las personas que tienen un elevado nivel de ansiedad ante la muerte padecen de un deterioro en su vida personal, laboral, interpersonal y afectiva, al igual que ocurre con los elevados niveles de ansiedad general (Mogotsi et al., 2000). Por otra parte, se ha detectado que las personas con altos niveles de ansiedad ante la muerte muestran menores niveles de autoeficacia y un locus de control más externo (So-Kum Tang et al., 2002).

\section{Medición de la Ansiedad ante la Muerte}

$\mathrm{Al}$ igual que con la ansiedad, la forma más común de medir este constructo es mediante autoinformes (Cano-Vindel \& Miguel-Tobal, 1990). Las ventajas de los autoinformes radican en que son técnicas de fácil aplicación e interpretación, con bajos costos económicos y que pueden aplicarse en múltiples ocasiones para ver cómo cambia una variable a través del tiempo (Hartung et al., 2011). Una gran variedad de instrumentos a este respecto se generó en la década de los setenta (Limonero García, 1997). Los primeros instrumentos de medición de la ansiedad ante la muerte fueron los publicados por Collett y Lester (1969) y Templer (1970), por lo cual, la ansiedad ante la muerte es una de las líneas de investigación más fructíferas dentro de la tanatología (Kastenbaum, 1988) y se ha evaluado respecto del sexo, edad, enfermedades crónicas, depresión, religión, personalidad, entre otros (Limonero García, 1997). Así, existen varias técnicas desarrolladas para medir la misma (Lester \& Castromayor, 1993; Neimeyer, 1994).

Templer (1970) desarrolló la Death Anxiety Scale (Escala de Ansiedad ante la Muerte, DAS) que consta de 15 ítems dicotómicos que proporcionan un puntaje total de ansiedad ante la muerte, la cual se ha convertido en uno de los instrumentos más empleados en el mundo a este respecto, mostrando buenas propiedades psicométricas tanto en población comunitaria como clínica (Miaja \& Moral, 2012). Dicha escala ha sido adaptada a distintos idiomas y culturas (Rivera-Ledesma \& Montero-López Lena, 2010). Entre los idiomas a los cuales se adaptó la Escala de Ansiedad ante la Muerte de Templer se encuentra el español (Tomás-Sábado \& Gómez-Benito, 2002, 2004), portugués, alemán, turco y árabe, entre otros idiomas (RiveraLedesma \& Montero-López Lena, 2010). A pesar de haber sido traducida al español, pocos son los estudios existentes sobre sus propiedades en los países de la América Latina (Rivera-Ledesma \& Montero-López Lena, 2010). Desarrollar y validar instrumentos que midan un importante constructo como la ansiedad ante la muerte es de un gran interés científico y terapéutico (Saleem et al., 2015), ya que la muerte es un hecho universal con importantes implicancias psicosociales.

\section{El Presente Estudio}

El propósito de este estudio consistió en la evaluación de las propiedades psicométricas de la Escala de Ansiedad ante la Muerte de Templer (1970) en una muestra de adultos emergentes de la Argentina. Debido 
a la importancia del constructo en cuestión, es fundamental evaluar las propiedades de un instrumento breve - el cual puede funcionar como un screening-, altamente reconocido a nivel mundial para evaluar la ansiedad ante la muerte en poblaciones comunitarias y no comunitarias. Así, la fortaleza del presente estudio radicó en ser el primero en Sudamérica en llevar a cabo una validación preliminar de dicha escala.

A partir de la problemática planteada, los objetivos fueron:

1. Observar la estructura factorial de la Escala Ansiedad ante la Muerte de Templer.

2. Determinar la consistencia interna (alfa de Cronbach y confiabilidad compuesta) del instrumento y la confiabilidad test/retest.

3. Explorar la validez de constructo.

\section{Método}

\section{Participantes}

Se seleccionó una muestra intencional no probabilística de $n=859$ adultos emergentes de Ciudad Autónoma de Buenos Aires y Gran Buenos Aires, Argentina. Los criterios de inclusión fueron: ser adulto emergente (edad entre 18 y 30), residir en las localidades señaladas y tener completados los estudios primarios. Se escogió a participantes adultos emergentes (Arnett, 2000, 2001) para evitar tomar muestras de personas en distintas etapas de la vida, como adolescentes o adultos mayores, ya que una alta heterogeneidad de la muestra - sea en edad u otra variable- puede afectar los estudios destinados a examinar la estructura factorial de un test (Tabachnick \& Fidell, 2013). En lo referente al sexo, 30\% eran varones y 70\% mujeres $(M=28,11, D E=11,20)$. Cuatro participantes tenían más de 30 años, por lo cual se los eliminó del análisis. Con relación al nivel educativo, 7\% no terminó la secundaria, 18\% terminó la secundaria, 39\% estaba cursando estudios terciarios/universitarios, $18 \%$ terminó estudios terciarios/universitarios y el resto terminó o estaba cursando estudios de posgrado, como doctorados o maestrías. El 35\% residía con sus progenitores, un $35 \%$, con pareja amorosa o hijos, $21 \%$, solo, $7 \%$, con amigos o compañeros y el grupo restante vivía con familiares que no eran sus padres (tíos, abuelos, entre otros). Un 15\% pertenecía a sectores socioeconómicos altos, un $71 \%$, a sectores medios y el restante era de sectores medio-bajos. Una submuestra de 250 participantes seleccionados al azar de los 859 participantes contestó dicha escala 6 meses después, con el fin de evaluar su confiabilidad test/retest.

\section{Instrumentos}

\section{Cuestionario Sociodemográfico ad Hoc}

Se preguntó edad, sexo, lugar de residencia, percepción de sector socioeconómico, entre otros.

\section{Escala de Ansiedad ante la Muerte de Templer (1970)}

La escala de Ansiedad ante la Muerte de Templer está constituida por 15 ítems con cuatro alternativas de respuesta con formato de respuesta tipo Likert: $1=$ Nunca o casi nunca, $2=$ Algunas veces, $3=$ La mayor parte del tiempo y $4=$ Todo el tiempo, siendo 15 el puntaje mínimo (mínima ansiedad) y 60 el puntaje máximo (máxima ansiedad). Algunos ítems de esta escala son: "Tengo miedo de morir", "Me molestan ciertos pensamientos sobre la muerte" y "Me da miedo tener una muerte dolorosa". Originalmente la escala de Ansiedad Ante la Muerte de Templer consistía de 15 ítems, con opción de respuesta de tipo verdadero o falso, de los cuales nueve recibían un punto si señalaba verdadero y los seis restantes recibían un punto si se contestaba falso. Posteriores estudios a los de Templer cambiaron las opciones dicotómicas por opciones contempladas en una escala Likert (López Castedo et al., 2004), como la aquí empleada. Este cambio se realizó con el fin de que los ítems tuvieran un mayor grado de variabilidad y discriminación (Hoogstraten et al., 1998; McMordie, 1979), lo cual brinda una mayor precisión y consistencia interna (Rivera-Ledesma \& MonteroLópez Lena, 2010). Los ítems para calcular los puntajes se pueden sumar o promediar, aunque es más común sumarlos.

Sus propiedades psicométricas están altamente comprobadas en los Estados Unidos y España, con alfa de Cronbach fluctuando entre 0,76 y 0,87 y una estabilidad test retest muy adecuada, $r=0,71$ y $r=0,84$ (López Castedo et al., 2004). En lo referente a su validez de constructo, la misma está altamente estudiada y se ha determinado su asociación con numerosas variables psicológicas, como depresión y ansiedad ( $r=0,38 \mathrm{y}$ $r=0,48$, respectivamente), con enfermedades físicas, como virus de inmunodeficiencia humana (HIV o cáncer 
(Lo et al., 2011; Rivera-Ledesma et al., 2010) y con otras escalas que miden el mismo constructo (López Castedo et al., 2004; Miaja \& Moral, 2012). En cambio, las investigaciones sobre su estructura factorial son más discrepantes a través de los estudios, las muestras y los contextos culturales en que se aplica. Por ejemplo, Templer (1970) postulaba una escala unifactorial, pero otros estudios en población hispana han detectado una estructura de dos a cinco factores (López Castedo \& Calle Santos, 2008; Miaja \& Moral, 2012; Rivera-Ledesma et al., 2010) en población comunitaria y clínica. La estructura de dos factores se integra por Miedo a la Muerte y Miedo a la Agonía/Enfermedad (Rivera-Ledesma et al., 2010), mientras que la de cinco factores se compone de Negación de la Muerte, Ansiedad ante la Muerte, Ansiedad Cognitiva y Fin de la Vida, Miedo al Dolor y Preocupación Generalizada ante la Muerte (López Castedo \& Calle Santos, 2008). Dichos factores están altamente asociados entre sí (Miaja \& Moral, 2012).

En el presente estudio se tomó como modelo la adaptación al español mexicano (Rivera-Ledesma \& Montero-López Lena, 2010), la cual presentó buenas propiedades psicométricas. En primer lugar, un docente de inglés hizo una traducción inversa al inglés de la adaptación mexicana de Rivera-Ledesma y MonteroLópez Lena (2010), mientras que otro juez independiente - experto en inglés - comprobó su equivalencia, al compararla con la original en inglés. En segundo lugar, tres jueces expertos independientes (dos recientes egresados de psicología y un psicólogo clínico experto en ansiedad) chequearon dicha traducción y sugirieron que solo en el ítem 6 ("Tengo miedo de tener cáncer") era adecuado agregar "u otro tipo de enfermedad grave". Como se consideró pertinente la modificación, se agregó dicha sugerencia. Antes de aplicar la escala a la muestra de la presente investigación, se aplicó en una muestra piloto de 40 sujetos de 18 a 40 años de edad de la Ciudad Autónoma de Buenos Aires, Argentina, debido a que era la primera vez que se aplicaba en este país. Cuando contestaron el test, se les dijo a los participantes que verbalizaran cualquier duda o inquietud. Los participantes señalaron que las preguntas eran claras y que no tenían dudas importantes. El alfa de Cronbach del estudio piloto fue 0,84 .

Para explorar la validez de constructo, se aplicaron las siguientes escalas:

\section{Escala de Depresión, Ansiedad y Estrés (Depression, Anxiety and Stress Scale DASS-21) de S. H. Lovibond y P. F. Lovibond (1995)}

Se trata de una medida de autoinforme que está constituida por subescalas que miden tres estados emocionales negativos, Depresión, Ansiedad y Estrés, con ítems como "Me resultaba difícil calmarme", "Todo me parecía negativo" y "Me parece que estaba al borde de un ataque de nervios", todos refiriéndose a los últimos 7 días. En el DASS-21 cada subescala cuenta con siete ítems. Los ítems constan de cuatro niveles de severidad/frecuencia que van desde no me pasaba (0) hasta me pasaba muchísimo (3), siendo los puntajes más altos los que indican un mayor nivel de emotividad negativa. Aquí no se describe la subescala Estrés, ya que no fue usada en el presente estudio. La subescala Ansiedad mide la activación del sistema nervioso autónomo y la experiencia subjetiva del afecto ansiedad. La subescala Depresión evalúa disforia, desesperanza, desinterés por la vida, anhedonia, autodesaprobación y desmotivación. Con relación a la consistencia interna, la escala posee un alfa de Cronbach de 0,81 para Depresión y 0,73 para Ansiedad (P. F. Lovibond \& S. H. Lovibond, 1995). Presenta, además, evidencia de validez factorial concurrente (por ejemplo, con las escalas Beck de Depresión y Ansiedad) y discriminante (P. F. Lovibond \& S. H. Lovibond, 1995). Fue adaptada a la Argentina por Facio et al. (2008), con buenas propiedades psicométricas.

\section{Inventario de Preocupación de Pensilvania (PSWQ) de Meyer et al. (1990)}

Este inventario evalúa la tendencia general a preocuparse o preocupación rasgo. Está compuesto por 16 ítems, cada uno con cinco alternativas de respuesta que van desde no me pasa (1) a me pasa mucho (5). Algunos de estos ítems son: "Se preocupa si no tiene suficiente tiempo para hacerlo todo", "Cuando está bajo tensión tiende a preocuparse mucho" y "Siempre está preocupándose por algo". Puntajes más altos implican mayor nivel del constructo medido. Este inventario presenta buena validez convergente y divergente, así como una elevada consistencia interna (alfa de Cronbach $=0,95$ ) en los Estados Unidos con valores similares hallados en muestras de otros países (Meyer et al., 1990). Con relación a la capacidad predictiva de la presencia del trastorno de ansiedad generalizada y su utilidad como medida en el campo terapéutico, este test ha recibido un amplio apoyo empírico (Meyer et al., 1990). Este inventario fue adaptado a la Argentina con buenas propiedades (Estherren, 2008). 


\section{Procedimiento}

La recolección de datos fue hecha durante los meses de octubre y noviembre de 2015. Para la recolección se contactó los participantes por teléfono, en las redes sociales o personalmente, si cumplían los criterios de inclusión de la muestra. El contacto por teléfono o redes sociales era simplemente para una primera aproximación al participante. En muchos casos, luego de una toma de datos, se le preguntaba si conocía a alguien que estuviera interesado en participar y, de responder afirmativamente, se le pedía algún medio para contactarlo, como un teléfono. En caso de que el participante manifestara estar interesado en colaborar, se acordaba un encuentro personal en el lugar que él designara (su casa, un bar, la facultad, entre otros). Los instrumentos fueron aplicados por los autores de este artículo y se tardó alrededor de 40 minutos en promedio en ser completados. Se aseguró el anonimato, la confidencialidad y la participación voluntaria en todo el proceso de recolección de datos mediante un consentimiento informado por escrito y firmado por los participantes. El estudio fue revisado y aprobado por el comité de ética de la Universidad Argentina de la Empresa.

\section{Análisis de Datos}

Los datos fueron procesados en el software estadístico SPSS para Windows (versión 22 en castellano). Para evaluar la estructura factorial de la escala de Ansiedad ante la Muerte de Templer, se utilizó el programa AMOS (Versión 16), llevándose a cabo un análisis factorial exploratorio con el procedimiento de máxima verosimilitud, debido a que los ítems presentaban una distribución relativamente normal, con una asimetría que iba de 0,41 a 2,16 y una curtosis que fluctuaba entre 0,09 a 4,55. Valores de asimetría mayores a 3 y de curtosis de 8 o más son considerados como extremos o críticos (Kline, 1998). Se establecieron autovalores mayores a 1 con rotación oblicua de Oblimin, suponiendo que, de emerger más de un factor, ambas dimensiones estarían altamente asociadas. Como la estructura factorial del test de ansiedad ante la muerte nunca se había explorado en las naciones de Sudamérica, se dividió la muestra en dos subgrupos aleatorios, uno de 400 participantes y otro de 459. Si bien los análisis factoriales exploratorios y confirmatorios pueden ser llevados a cabo en una misma muestra, lo deseable es emplear dos muestras independientes (Hurley et al., 1997). Se tomó a la primera muestra como calibración y a la segunda como replicación. Por dicho motivo, se decidió comenzar con un análisis factorial exploratorio, para luego llevar a cabo un análisis confirmatorio. La estructura factorial del test de Templer en muestras hispanas presenta una gran discrepancia, debido a la inconsistencia de los estudios en la cantidad de dimensiones de la escala (por ejemplo, López Castedo \& Calle Santos, 2008; Miaja \& Moral, 2012), por lo cual se buscó explorar en la primera muestra para confirmar en la segunda, ya que las hipótesis sobre la estructura factorial de un constructo pueden emerger de la literatura científica o de estudios empíricos. Ambas técnicas son útiles y pueden complementarse (Hurley et al., 1997).

Para determinar si el ajuste del modelo en el análisis factorial confirmatorio era adecuado, se tuvieron en cuenta el índice comparativo de Bentler-Bonett (CFI), el índice de ajuste relativo (RFI) y la raíz del residuo cuadrático medio estandarizado (SRMR), siguiendo las recomendaciones de Byrne (2010). No se consideró $\chi^{2}$, ya que se ha señalado que es un criterio muy exigente, por lo cual no se toma en cuenta (Byrne, 2010). Sin embargo, se sugiere dividir $\chi^{2}$ por los grados de libertad. Aunque no hay un consenso a este respecto, un valor por debajo de 2 es considerado satisfactorio (Cupani, 2012). Respecto de CFI y RFI, se sabe que los mismos deben estar por encima de 0,90 (Bentler, 1992) —o incluso 0,95, según criterios más actuales (Byrne, 2010; Hu \& Blenter, 1999)_, mientras que el SRMR debe ser menor a 0,10 o incluso a 0,05 (Byrne, 2010) para considerar un buen ajuste del modelo.

Para evaluar la confiabilidad test/retest y la validez de constructo se calcularon correlaciones de Pearson entre los puntajes de las distintas escalas. La muestra total de 859 de adultos emergentes se usó para evaluar la validez de constructo, para lo cual se calculó la correlación $r$ de Pearson entre los puntajes totales de la Escala de Ansiedad ante la Muerte de Templer y la Subescala de Ansiedad y Depresión del DASS-21 y el Inventario de Preocupación de Pensilvania.

\section{Resultados}

\section{Estructura Factorial}

Con respecto al primer objetivo de evaluar la estructura factorial de la escala de Ansiedad ante la Muerte de Templer, el análisis factorial exploratorio se realizó con la primera submuestra de 400 participantes. El 
$\mathrm{KMO}=0,83$ y el $\chi^{2}(105, n=400)=2711,34, p<0,001$ indicaron que era adecuado llevarlo a cabo. En la Tabla 1 se presentan los resultados de dicho análisis factorial. Como se ve ahí, emergieron dos factores, los cuales explicaban un $42 \%$ de la varianza de ansiedad ante la muerte. El primer factor explicaba un $29 \%$ de la varianza y el segundo, un 13\%. El primero se denominó Miedo a la Muerte y el segundo, Miedo a la Agonía/Fin de la Vida, siguiendo las conceptualizaciones de Rivera-Ledesma et al. (2010). Todos los ítems tenían una buena resolución factorial y cargaban por encima de 0,30, con excepción del ítem 14, que cargaba casi por igual en ambos factores. Por otra parte, también el ítem 15 tenía muy baja carga — por debajo de 0,20 - pero puntuaba en mayor medida en la subescala Miedo a la Agonía/Fin de la Vida que en el primer factor. Como se estaba en un proceso de validación inicial del test, se decidió conservarlos para observar cómo funcionaban psicométricamente en los restantes análisis.

En la Tabla 2 se presentan los estadísticos descriptivos para las dos subescalas y para el puntaje total de la escala de Templer. La correlación entre ambas subescalas o dimensiones fue $r(398)=0,47, p<0,001$.

Tabla 1

Cargas Factoriales de los Ítems de la Escala de Ansiedad ante la Muerte de Templer

\begin{tabular}{|c|c|c|}
\hline \multirow{2}{*}{ Ítem } & \multicolumn{2}{|c|}{ Componente } \\
\hline & Miedo muerte & Miedo agonía/fin de la vida \\
\hline 1. Tengo mucho miedo de morirme & 0,84 & \\
\hline 2. Pienso en la muerte & 0,82 & \\
\hline 3. Me pone nervioso que la gente hable de la muerte & 0,69 & \\
\hline $\begin{array}{l}\text { 4. Me asusta mucho pensar que tuvieran que } \\
\text { operarme }\end{array}$ & 0,65 & \\
\hline 5. Tengo miedo de morir & 0,66 & \\
\hline 6. Siento miedo de la posibilidad de tener cáncer & 0,53 & \\
\hline 7. Me molestan ciertos pensamientos sobre la muerte & 0,51 & \\
\hline 8. A menudo me preocupa lo rápido que pasa el tiempo & 0,45 & \\
\hline 9. Me da miedo tener una muerte dolorosa & 0,34 & \\
\hline 10. Me preocupa mucho el tema de la otra vida & & 0,81 \\
\hline $\begin{array}{l}\text { 11. Me asusta la posibilidad de sufrir un ataque al } \\
\text { corazón }\end{array}$ & & 0,79 \\
\hline 12. Pienso que la vida es muy corta & & 0,57 \\
\hline $\begin{array}{l}\text { 13. Me asusta oír hablar a la gente de una tercera } \\
\text { guerra mundial }\end{array}$ & & 0,48 \\
\hline 14. Me horroriza ver un cadáver & 0,33 & 0,39 \\
\hline 15. Pienso que tengo motivos para temer el futuro & & 0,17 \\
\hline
\end{tabular}

Nota. Se muestran las cargas factoriales por encima de 0,30, con la excepción del ítem 15. $n=400$ 
Tabla 2

Estadísticos Descriptivos de la Escala Ansiedad ante la Muerte de Templer

\begin{tabular}{lcccc}
\hline \multicolumn{1}{c}{ Escala/subescala } & Mín & Máx & $M$ & $D E$ \\
\hline Escala total de ansiedad ante la muerte & 10,00 & 40,00 & 17,45 & 3,87 \\
Miedo muerte & 9,00 & 36,00 & 15,02 & 4,01 \\
Miedo agonía/fin vida & 6,00 & 24,00 & 12,16 & 2,83 \\
\hline
\end{tabular}

$n=400$

Siguiendo con el segundo objetivo, se llevó a cabo un análisis factorial confirmatorio en la muestra de 459 participantes para determinar un modelo unifactorial —el del autor de la escala-y compararlo con un modelo bifactorial — siguiendo la estructura del modelo encontrado aquí- con el fin de determinar cuál poseía un mejor ajuste. Para el modelo bifactorial, al ítem 14 "Me horroriza ver un cadáver" se lo especificó cargando en ambos factores, como había emergido en el análisis factorial exploratorio.

Como se ve en la Tabla 3, el modelo unifactorial, denominado Modelo 1, no arrojó un buen ajuste: los índices de ajustes fueron muy inadecuados.

Al llevar a cabo un nuevo análisis para poner a prueba un modelo bifactorial, se halló un modelo (Modelo 2) con un ajuste satisfactorio, como se ve en la Tabla 3. Este ajuste se encontraba muy cercano a criterios más actuales y exigentes de CFI mayor de 0,97 y SRMR menor de 0,07 (Hair et al., 2010). Del mismo modo, el $\Delta \chi^{2}$ era estadísticamente significativo con respecto al modelo unifactorial, lo cual indicaba un mejor ajuste del modelo bifactorial. Asimismo, al dividir $\chi^{2}$ por los grados de libertad, se obtenía un valor aceptable de 1,62.

En la Tabla 4 se muestran las cargas factoriales del modelo bifactorial de Ansiedad ante la Muerte de Templer. Nuevamente, la pregunta 14 tuvo una baja carga factorial. La covarianza entre ambos factores fue $r(457)=0,66, p<0,001$.

\section{Confiabilidad}

En lo relativo al segundo objetivo de examinar la consistencia interna de la escala de Ansiedad ante la Muerte de Templer, alfa de Cronbach mostró un valor de 0,80 para la subescala Miedo a la Muerte y uno de 0,70 para la de Miedo a la Agonía/Fin de la Vida, mientras arrojó un alfa de 0,80 para la escala total — se calculó también la consistencia total debido a la alta correlación de los dos factores-. Al hacer un análisis más profundo de la consistencia interna de la escala y las dos subescalas, se observó que la mayoría de los ítems se hallaba por encima de 0,30 en la correlación ítem-test, con la excepción del ítem 14, tanto para las dimensiones Miedo a la Muerte y Agonía/Fin de la Vida como para la escala total. Por otra parte, el ítem 15 "Pienso que tengo motivos para temer el futuro" tuvo una correlación de 0,29 con el resto de los ítems. Al eliminar el ítem 14, la consistencia ascendía a 0,82, 0,71 y 0,83, respectivamente, por lo cual se decidió eliminarlo para los restantes análisis. La consistencia interna no mejoraba al eliminar el ítem 15 y, debido a que su valor estaba muy cercano de 0,30 se decidió conservarla. Los valores de la consistencia interna compuesta fueron 0,89 para la subescala Miedo a la Muerte, 0,71 para Miedo a la Agonía/Fin de la Vida y 0,91 para la escala total.

La confiabilidad test/retest para el subgrupo de los 250 participantes que contestaron la escala 6 meses después fue $r(248)=0,66$ para la escala total y $r(248)=0,68$ y $r(248)=0,63$ para Miedo a la Muerte y Miedo a la Agonía/Fin de la Vida, respectivamente, con una significación de $p<0,01$.

\section{Validez de Constructo}

Con respecto al tercer objetivo de evaluar la validez de constructo de la Escala de Ansiedad de Templer en la muestra total $(n=859)$, se hallaron relaciones significativas de tamaño mediano entre el puntaje total de la escala de Templer y los puntajes de preocupación, ansiedad y depresión. Como se ve en la Tabla 5, la ansiedad ante la muerte correlacionó con ansiedad, depresión y preocupación. Para las dos subescalas de ansiedad ante la muerte, también hubo correlaciones. Todas las asociaciones eran de tamaño mediano - estaban entre 0,30 y 0,50 - con la excepción de los puntajes de miedo a la muerte y depresión que se hallaba por debajo de 0,30 (Cohen, 1988). 
Tabla 3

Ajustes de los Modelos de la Escala de Ansiedad ante la Muerte de Templer

\begin{tabular}{lccccccc}
\hline \multicolumn{1}{c}{ Modelo } & $\chi^{2}$ & $g l$ & CFI & RFI & SRMR & $\Delta \chi^{2}$ & $\Delta g l$ \\
\hline $\begin{array}{l}\text { Modelo 1 } \\
\text { unifactorial }\end{array}$ & 687,14 & 90 & 0,73 & 0,69 & 0,10 & & \\
$\begin{array}{l}\text { Modelo 2 } \\
\text { bifactorial }\end{array}$ & 144,22 & 89 & 0,97 & 0,92 & 0,03 & $542,92^{* * *}$ & 1 \\
\hline
\end{tabular}

Nota. ${ }^{* *} p<0,001, g l=$ grados de libertad, CFI = índice comparativo de Bentler-Bonett, RFI = índice de ajuste relativo, $\mathrm{SRMR}=$ raíz del residuo cuadrático medio estandarizado, $\Delta \chi^{2}=$ diferencia de $\chi^{2}, \Delta g l=$ diferencia entre los grados de libertad. $n=459$.

Tabla 4

Cargas Factoriales de los Items de la Escala de Ansiedad ante la Muerte de Templer del Modelo Bifactorial

\begin{tabular}{lll}
\hline \multicolumn{1}{c}{ Ítem } & Carga & Carga \\
\hline 1. Tengo mucho miedo de morirme & $0,77^{* * *}$ & \\
2. Pienso en la muerte & $0,51^{* * *}$ & \\
3. Me pone nervioso que la gente hable de la muerte & $0,57^{* * *}$ & \\
4. Me asusta mucho pensar que tuvieran que operarme & $0,38^{* * *}$ & \\
5. Tengo miedo de morir & $0,70^{* * *}$ & $0,43^{* * *}$ \\
6. Siento miedo de la posibilidad de tener cáncer & $0,55^{* * *}$ & \\
7. Me molestan ciertos pensamientos sobre la muerte & $0,54^{* * *}$ & \\
8. A menudo me preocupa lo rápido que pasa el tiempo & $0,53^{* * *}$ & \\
9. Me da miedo tener una muerte dolorosa & & $0,44^{* * *}$ \\
10. Me preocupa mucho el tema de la otra vida & & $0,47^{* * *}$ \\
11. Me asusta la posibilidad de sufrir un ataque al corazón & & $0,54^{* * *}$ \\
12. Pienso que la vida es muy corta & $0,14^{* * *}$ & $0,28^{* * *}$ \\
13. Me asusta oír hablar a la gente de una tercera guerra mundial & & $0,15^{* *}$ \\
14. Me horroriza ver un cadáver & & $0,48^{* * *}$ \\
15. Pienso que tengo motivos para temer el futuro & \\
\hline
\end{tabular}

${ }^{* * *} p<0,001,{ }^{* *} p<0,05, n=459$.

Tabla 5

Correlaciones de Pearson entre el Puntaje Total de Ansiedad ante la Muerte, Miedo a la Muerte, Miedo a la Agonía /Fin de la Vida y Ansiedad, Depresión y Preocupación

\begin{tabular}{lccc}
\hline Escala/subescala & Ansiedad ante la muerte & Miedo a la muerte & Agonía/fin de la vida \\
\hline Preocupación & $0,45^{* * *}$ & $0,38^{* * *}$ & $0,40^{* * *}$ \\
Ansiedad & $0,48^{* * *}$ & $0,41^{* * *}$ & $0,42^{* * *}$ \\
Depresión & $0,39^{* * *}$ & $0,28^{* * *}$ & $0,40^{* * *}$ \\
\hline
\end{tabular}

${ }^{* * *} p<0,001 . n=859$

\section{Discusión}

En lo relativo al primer objetivo de la investigación, al realizar un análisis factorial exploratorio emergió una estructura factorial de dos dimensiones, en lugar de un factor, como postulaba el autor de dicho instrumento. El primer factor podía ser denominado Miedo a la Muerte y el segundo, Miedo a la Agonía/Fin de la Vida. Cabe aclarar que los estudios de Templer (1970) son de la década de los 70 y, desde entonces, 
muchos cambios sociales, culturales y económicos se han producido en lo referente a las actitudes ante la muerte. Un estudio en México con población comunitaria también halló una estructura de dos factores que explicaba un 57\% de la varianza de ansiedad ante la muerte (Rivera-Ledesma et al., 2010), por lo cual, los resultados aquí encontrados fueron similares. Sin embargo, otros trabajos en países hispanos detectaron tres o cuatro factores y hasta cinco (López Castedo \& Calle Santos, 2008; Miaja \& Moral, 2012), aunque el estudio de López Castedo y Calle Santos (2008) trabajó con población clínica, con pacientes con HIV. Como la ansiedad ante la muerte es un constructo matizado por factores sociales, psicológicos, históricos y culturales, es posible que el contexto y la idiosincrasia introduzcan diferencias en cómo se percibe dicha variable. Por su parte, Tomás-Sábado y Gómez-Benito (2004) sugieren que la escala es susceptible a las diferencias culturales con respecto al concepto de muerte, por lo cual su estructura factorial puede variar de acuerdo a cada región (Beshai \& Naboulsi, 2004; Lester, 1993). De allí que las discrepancias con respecto a sus propiedades psicométricas son esperables (Hoogstraten et al., 1998), como lo indican las diferentes estructuras factoriales halladas a través de las investigaciones en distintos contextos culturales. Así, los resultados de este trabajo son coincidentes con los de las investigaciones que señalan la multidimensionalidad del constructo (Lonetto \& Templer, 1986).

El por qué varía su estructura en los distintos países o culturas debe seguir siendo examinado, pero probablemente se deba a que la muerte no es un hecho simple ni instantáneo. También las distintas estructuras factoriales pueden deberse a las diferentes metodologías y poblaciones examinadas: muestras intencionales, población clínica versus comunitaria, por ejemplo.

Con respecto a los niveles de ansiedad ante la muerte, la media en la presente investigación es muy similar a las detectadas en otras investigaciones (por ejemplo, Rivera-Ledesma et al., 2010). Cabe aclarar que los estudios de Templer empleaban alternativas dicotómicas, dando lugar a un puntaje máximo de 15, lo cual no permite hacer comparaciones con los presentes resultados que empleó una escala de respuesta tipo Likert, como hicieron investigaciones posteriores.

Todos los ítems tenían una buena carga factorial y, en general, no había cargas cruzadas, con excepción del ítem 14 ("me horroriza ver un cadáver"). También el estudio de Rivera-Ledesma et al. (2010) halló que este ítem presentaba dificultades y no era un buen indicador de la ansiedad ante la muerte. Es probable que a muchas personas les horroriza o les desagrada ver un cadáver, por asco o impresión, y no porque tengan ansiedad ante la muerte. Todas las preguntas tuvieron una muy buena carga factorial en el modelo de dos factores, con la sola excepción del ítem señalado, el cual se había especificado cargando en ambos factores. Esto señala, nuevamente, que dicho ítem no es un buen indicador del constructo. Este ítem y el que señala "Pienso que tengo motivos para temer el futuro" deberían ser examinados en futuros estudios exploratorios, como en grupos focales y entrevistas, con el fin de examinar por qué presentan tan baja carga factorial, en comparación con el resto, y poder ser reelaborados. Esto es, examinar en detalle cuál es el significado que para las personas tiene "me horroriza ver un cadáver" y "pienso que tengo motivos para temer el futuro". ¿Es ver un cadáver un hecho o pensamiento infrecuente? ¿genera asco o repulsión antes que ansiedad ante la muerte? Por otra parte, es posible también que ver un cadáver sea un hecho infrecuente. Como se señaló, Rivera-Ledesma et al. (2010) también detectaron dificultades con este reactivo al tener una muy baja carga factorial. Estudios exploratorios podrían aportar elementos para reescribir dicho ítem o para ser sustituido por otro, del mismo modo que el otro ítem comentado. En este último caso, es posible que las respuestas estuvieran afectadas por el contexto de inestabilidad política y económica tan frecuente en la Argentina, lo cual explicaría su baja carga factorial. Sin embargo, más investigación es necesaria al respecto con muestras de otras regiones de la Argentina y de otras naciones de Sudamérica, antes de decidir eliminar el ítem 15 de forma definitiva, modificarlo o sustituirlo.

En lo relativo al segundo objetivo, la consistencia interna fue adecuada para la dimensión miedo a la muerte y para miedo agonía/fin vida, como para la escala total, ya que valores por encima de 0,70 se consideran correctos (DeVellis, 2012; Kaplan \& Saccuzzo, 2006). Los estudios informan alfas de Cronbach de la escala entre 0,76 y 0,87 (López Castedo \& Calle Santos, 2008; Templer, 1970; Tomás-Sábado \& GómezBenito, 2002), por lo tanto, los hallazgos aquí detectados se hallaron dentro de dichos parámetros, con la excepción de Miedo a la Agonía/Fin de la Vida. La confiabilidad compuesta — un indicador moderno y que no está afectado por la cantidad de ítems - también fue satisfactoria, ya que para las dos subescalas y para la escala total se hallaba por encima de 0,70, como sugiere Hair et al. (1995). En lo referente a la confiabilidad test/retest, estas fueron similares a las detectadas en otros estudios (López Castedo \& Calle Santos, 2008). 
En lo relativo al tercer objetivo, se halló validez de constructo con ansiedad, depresión y preocupación, con asociaciones medianas en todos los casos, con la única excepción de los puntajes de depresión y miedo a la muerte. Esto concuerda con el estudio de Rivera-Ledesma et al. (2010), el cual también halló correlaciones del mismo tamaño con depresión y ansiedad. Que las correlaciones fuesen de tamaño mediano es una prueba de una adecuada validez, ya que de ser altas indicaría que se estaría evaluando el mismo constructo (Cohen, 1988). Por su parte, un estudio que también usó la escala de Templer encontró correlaciones con la ansiedad, pero demostrando que la ansiedad ante la muerte y la ansiedad eran constructos diferenciados (TomásSábado et al., 2007).

Los resultados indicarían que la Escala de Ansiedad ante la Muerte de Templer en su versión preliminar presentaría buenas propiedades psicométricas en la Argentina, eliminando el ítem "Me horroriza ver un cadáver". El hecho de que no se replique el modelo unifactorial de Templer puede deberse no solo a factores sociales, culturales e históricos, sino también a que en el presente estudio se introdujo la versión tipo Likert, la cual brinda una mayor sensibilidad y discriminación (Rivera-Ledesma \& Montero-López Lena, 2010). Sin embargo, estos resultados son alentadores al arrojar evidencias de que una versión preliminar de la escala de Templer mostraría validez y confiabilidad en la Argentina para ser usada en esta versión de 14 preguntas.

Se sabe que la muerte no es un hecho solamente biológico, sino que está atravesado por factores psicosociales, culturales y personales, lo cual genera intensas emociones en las personas. De este modo, una enfermedad crónica o terminal puede agravarse si el paciente sufre de una gran ansiedad o emociones negativas en un alto nivel, como ira o tristeza. En este sentido dicha escala - al ser un autoinforme breve, pero válido y confiable - puede ser empleada como un rápido screening para detectar personas con alta ansiedad ante la muerte y realizar tareas de prevención e intervención para mejorar la salud mental de los pacientes terminales o con enfermedades crónicas. También puede ser una escala útil en otras poblaciones, como adultos mayores hospitalizados o que residan en geriátricos, quienes pueden experimentar altos niveles de ansiedad ante la muerte y agravar su salud física o mental. De este modo, el valor del presente estudio no es solamente teórico o con fines de investigación básica, sino que tiene implicancias prácticas y terapéuticas. En la misma línea, se podría ayudar a estos pacientes, como a sus familiares, a analizar sus actitudes hacia la muerte, las emociones que despierta la misma y tratar de valorar la vida, como sugiere Carrobles, Remor y Rodríguez-Alzamora (2003). Aunque la persona esté padeciendo una enfermedad grave, se debe valorar la vida, pero también se debe educar en que la muerte es un proceso natural y biológico, sin dejar de lado los aspectos sociales, culturales, espirituales y psicológicos involucrados en la misma. La brevedad de la escala es una gran ventaja, considerando que instrumentos más extensos pueden ser intrusivos o estresantes en poblaciones vulnerables, como pacientes que sufren enfermedades físicas o adultos mayores.

Una limitación del presente trabajo fue la muestra, la que fue conformada de manera intencional no probabilística, lo que no permite generalizar los resultados a toda la población. Además, se componía de más mujeres que varones. Otra limitación es que los datos se recolectaron por autoinforme, el cual presenta reconocidas limitaciones, como la subjetividad al responder, deseabilidad social, falta de honestidad, entre otras. Asimismo, se usó el autoinforme para medir las restantes variables, lo que aumenta artificialmente las relaciones entre las variables, debido a la varianza compartida por el método de recolección de datos. Otra limitación es que se trataba de un estudio correlacional y transversal, por lo cual no se puede observar cómo cambia el fenómeno a través del tiempo — al margen de que se haya examinado su confiabilidad test/retest en un subgrupo- o inferir la direccionalidad de la causalidad entre las variables: ¿es el afecto negativo el que influye en la ansiedad ante la muerte? $\mathrm{O}$ ¿la ansiedad ante la muerte influye en el afecto negativo? O ¿existen relaciones bidireccionales entre las variables?

Futuros estudios deberían trabajar con muestras aleatorias, de diversas edades, por ejemplo, adultos jóvenes, adultos mayores, de diferentes estratos sociales, de distintas regiones de la Argentina y de otros países de la región, para seguir examinando las propiedades de la escala, principalmente su estructura factorial. Del mismo modo, sería interesante ver las propiedades del instrumento en población clínica, como en pacientes hospitalizados por enfermedades físicas crónicas o terminales y personas con trastornos mentales. Sería adecuado, también, observar la invarianza del instrumento en distintas naciones de Latinoamérica y en población clínica versus comunitaria. De igual manera, se debería estudiar la validez convergente de esta escala con otro instrumento que mida el mismo constructo, como el Inventario de Ansiedad ante la Muerte de Tomás-Sábado y Gómez-Benito (2005). Asimismo, se deberían incluir otros informantes, como amigos o parejas, para evitar las limitaciones del autoinforme. Por otra parte, sería interesante observar el funcionamiento de los ítems con la teoría de respuesta al ítem. Además, se debería 
llevar a cabo un estudio longitudinal y con muestras de mayor tamaño para determinar la confiabilidad test/retest y para examinar la direccionalidad de la causalidad entre la ansiedad ante la muerte y otras variables psicológicas relevantes. Finalmente, un paso importante sería explorar la validez de criterio con otros indicadores, como diagnósticos de enfermedades físicas, como HIV o cáncer.

\section{Referencias}

Arnett, J. J. (2000). Emerging adulthood: A theory of development from the late teens through the twenties. American Psychologist, 55(5), 469-480. https://doi.org/10.1037/0003-066X.55.5.469

Arnett, J. J. (2001). Conceptions of the transition to adulthood: Perspectives from adolescence through midlife. Journal of Adult Development, 8(2), 133-143. https://doi.org/10.1023/A:10264013225

Bentler, P. M. (1992). On the fit of models to covariances and methodology to the Bulletin. Psychological Bulletin, 112(3), 400404. https://doi.org/10.1037/0033-2909.112.3.400

Beshai, J. A. \& Naboulsi, M. A. (2004). Existential perspectives on death anxiety. Psychological Reports, 95(2), 507-513. https://doi.org/10.2466/pr0.95.2.507-513

Byrne, B. M. (2010). Structural equation modeling with AMOS: Basic concepts, applications, and programming (2a ed.). Routledge. https://doi.org/10.4324/9780203726532

Cano-Vindel A. \& Miguel-Tobal J. J. (1990). Evaluación mediante autoinforme de la reactividad cognitivo-físiológica-motora ante diferentes situaciones: diferencias individuales y situacionales. En Colegio Oficial de Psicólogos de Madrid (Ed.), Psicología española en la Europa de los 90. Ciencia y profesión. Volumen 9: A. Ávida Espada (Coord.), Diagnóstico y evaluación psicológica (pp. 94-101).. https://www.researchgate.net/publication/230577070_Evaluacion_mediante_autoinforme_de_la_reactividad_cognitivo-fisiologicamotora ante diferentes situaciones Diferencias individuales y situacionales Assessment by self-report of the cognitive-physiolog

Carrobles, J. A., Remor, E. \& Rodríguez-Alzamora, L. (2003). Afrontamiento, apoyo social percibido y distrés emocional en pacientes con infección por VIH. Psicothema, 15(3), 420-426. http://www.psicothema.com/pdf/1082.pdf

Cohen, J. (1988). Statistical power analysis for the behavioral sciences (2a ed.). Lawrence Erlbaum.

Collett, L. -J. \& Lester, D. (1969). The fear of death and the fear of dying. The Journal of Psychology, 72(2), 179-181. https://doi.org/10.1080/00223980.1969.10543496

Cupani, M. (2012). Análisis de ecuaciones estructurales: conceptos, etapas de desarrollo y un ejemplo de aplicación. Revista Tesis, 2, 186-199. https://revistas.unc.edu.ar/index.php/tesis/article/view/2884

DeVellis, R. F. (2012). Scale development: Theory and applications (4a ed.). SAGE.

Estherren, L (2008). La ansiedad y la relación con la preocupación y la incertidumbre en adultos de Paraná [Tesis de grado no publicada]. Universidad Católica Argentina.

Facio, A., Micocci, F. \& Resett, S. (2008). Haciéndose adulto: relaciones interpersonales y problemas emocionales y de conducta en la adultez emergente (Proyecto de investigación y desarrollo 171/08). Universidad Nacional de Entre Ríos, Facultad de Ciencias de la Educación.

Grau Abalo, J., Llantá Abreu, M. C., Massip Pérez, C., Chacón Roger, M., Reyes Méndez, M. C., Infante Pedreira, O. \& Morales Arisso, D. (2008). Ansiedad y actitudes ante la muerte: revisión y caracterización en un grupo heterogéneo de profesionales que se capacita en cuidados paliativos. Pensamiento Psicológico, 4(10), 27-58. https://www.redalyc.org/pdf/801/80111670003.pdf

Hair Jr., J. F., Anderson, R. E., Tatham, R. L. \& Black, W. C. (1995). Multivariate data analysis. Prentice Hall.

Hair Jr., J. F., Black, W. C., Babin, B. J. \& Anderson, R. E. (2010). Multivariate data analysis. Seventh Edition. Prentice Hall.

Hartung, C. M., Little, C. S., Allen, E. K. \& Page, M. C. (2011). A psychometric comparison of two self-report measures of bullying and victimization: Differences by sex and grade. School Mental Health, 3(1), 44-57. https://doi.org/10.1007/s12310-010-9046-1

Hoogstraten, J., Koele, P. \& Van der Laan, J. (1998). Templer's Death Anxiety Scale Revisited: The Dutch version. Perceptual and Motor Skills, 87(3_suppl), 1259-1264. https://doi.org/10.2466/pms.1998.87.3f.1259

Hu, L. -t. \& Bentler, P. M. (1999). Cutoff criteria for fit indexes in covariance structure analysis: Conventional criteria versus new alternatives. Structural Equation Modeling, 6(1), 1-55. https://doi.org/10.1080/10705519909540118

Hurley, A. E., Scandura, T. A., Schriesheim, C. A., Brannick, M. T., Seers, A., Vandenberg, R. J. \& Williams, L. J. (1997). Exploratory and confirmatory factor analysis: Guidelines, issues, and alternatives. Journal of Organizational Behavior, 18(6), 667-683. https://doi.org/10.1002/(SICI)1099-1379(199711)18:6<667::AID-JOB874>3.0.CO;2-T

Kaplan, R. M. \& Saccuzzo, D. P. (2006). Pruebas psicológicas: principios, aplicaciones y temas (6a ed.). Thomson.

Kastenbaum, R. (1988). Theory, research, and application: Some critical issues for thanatology. Omega: Journal of Death and Dying, 18(4), 397-410. https://doi.org/10.2190/3Y8A-ACF4-1XT9-NLDD

Kline, R. B. (1998). Software review: Software programs for structural equation modeling: AMOS, EQS, and LISREL. Journal of Psychoeducational Assessment, 16(4), 343-364. https://doi.org/10.1177/073428299801600407

Lehto, R. H. \& Stein, K. F. (2009). Death anxiety: An analysis of an evolving concept. Research and Theory for Nursing Practice, 23(1), 23-41. https://doi.org/10.1891/1541-6577.23.1.23

Lester, D. (1993). The structure of Templer's Death Anxiety Scale among Egyptian students: A comment. Psychological Reports, 72(3_suppl), 1378. https://doi.org/10.2466/pr0.1993.72.3c.1378

Lester, D. \& Castromayor, I. (1993). The construct validity of Templer's Death Anxiety Scale in Filipino students. The Journal of Social Psychology, 133(1), 113-114. https://doi.org/10.1080/00224545.1993.9712125

Limonero García, J. T. (1997). Ansiedad ante la muerte. Ansiedad y Estrés, 3(1), 37-48. http://suicidioprevencion.cienciassociales.edu.uy/wpcontent/uploads/2015/09/Ansiedad-ante-la-muerte.-Limonero.1997.pdf

Limonero García, J. T., Tomás-Sábado, J., Fernández Castro, J., Cladellas Pros, R. \& Gómez Benito, J. (2010) Competencia personal percibida $\mathrm{y}$ ansiedad ante la muerte en estudiantes de enfermería. Ansiedad y Estrés, 16(2-3), 177-188. https://dialnet.unirioja.es/servlet/articulo?codigo=3341071

Lo, C., Hales S., Zimmermann, C., Gagliese, L., Rydall, A. \& Rodin, G. (2011). Measuring death-related anxiety in advanced cancer: Preliminary psychometrics of the Death and Dying Distress Scale. Journal of Pediatric Hematology/Oncology, 33(Supplement 2), S140-S145. https://doi.org/10.1097/MPH.0b013e318230e1fd

Lonetto, R. \& Templer, D. I. (1986). Series in health psychology and behavioral medicine. Death anxiety. Hemisphere. 
López Castedo, A. \& Calle Santos, I. (2008). Características psicométricas de la Escala de Ansiedad ante la Muerte (DAS) en pacientes de VIH/SIDA. Psicothema, 20(4), 958-963. http://www.psicothema.com/psicothema.asp?id=3582

López Castedo, L., Sueiro Domínguez, E. \& López García, M. C. (2004). Ansiedad ante la muerte en la adolescencia. Revista GalegoPortuguesa de Psicoloxía e Educación, 11, 241-254. https://dialnet.unirioja.es/servlet/articulo?codigo=1038625

Lovibond, P. F. \& Lovibond, S. H. (1995). The structure of negative emotional states: Comparison of the Depression Anxiety Stress Scales (DASS) with the Beck Depression and Anxiety Inventories. Behaviour Research and Therapy, 33(3), 335-343. https://doi.org/10.1016/0005-7967(94)00075-U

Lovibond, S. H. \& Lovibond, P. F. (1995). Manual for the Depression Anxiety Stress Scales. Psychology Foundation of Australia.

McMordie, W. R. (1979). Improving measurement of death anxiety. Psychological Reports, 44(3), 975-980. https://doi.org/10.2466/pr0.1979.44.3.975

Meyer, T. J., Miller, M. L., Metzger, R. L. \& Borkovec, T. D. (1990). Development and validation of the Penn State Worry Questionnaire. Behaviour Research and Therapy, 28(6), 487-495. https://doi.org/10.1016/0005-7967(90)90135-6

Miaja, M. \& Moral, J. (2012). Propiedades psicométricas de la Escala de Ansiedad ante la Muerte en personas con VIH y población general. Revista de Psicopatología y Psicología Clínica, 17(2), 107-121. https://doi.org/10.5944/rppc.vol.17.num.2.2012.11208

Mogotsi, M, Kaminer, D. \& Stein, D. J. (2000). Quality of life in the anxiety disorders. Harvard Review of Psychiatry, 8(6), $273-282$. https://doi.org/10.1080/hrp.8.6.273

Neimeyer R. A. (Ed.) (1994). Death anxiety handbook: Research, instrumentation, and application. Taylor \& Francis.

Neimeyer, R. A. (Ed.) (2015). Death anxiety handbook: Research, instrumentation, and application (2a ed.). Routledge.

Rivera-Ledesma, A. \& Montero-López Lena, M. (2010). Propiedades psicométricas de la Escala de Ansiedad ante la Muerte de Templer en sujetos mexicanos. Diversitas: Perspectivas en Psicología, 6(1), 135-140. https://doi.org/10.15332/s1794-9998.2010.0001.10

Rivera-Ledesma, A, Montero-López Lena, M. \& Sandoval Ávila, R. (2010). Escala de Ansiedad ante la Muerte de Templer: propiedades psicométricas en pacientes con insuficiencia renal crónica terminal. Journal of Behavior, Health \& Social Issues, 2(2), 83-91. https://doi.org/10.5460/jbhsi.v2.2.26795

Saleem, T., Gul, S. \& Saleem, S. (2015). Death Anxiety Scale: Translation and validation in patients with cardiovascular $\begin{array}{lllll}\text { disease. The Medical } & \text { Pofessional } & \text { Journal, } & \text { 22(6), }\end{array}$ https://www.researchgate.net/publication/287865943_Death_Anxiety_Scale_Translation_Validation_in_Patients_with_Cardiovascular_disease

Sevilla-Casado, M. \& Ferré-Grau, C. (2013). Ansiedad ante la muerte en enfermeras de atención sociosanitaria: datos y significados. GEROKOMOS, 24(3), 109-114. https://doi.org/10.4321/S1134-928X2013000300003

So-Kum Tang, C., Wu, A. M. S. \& Yan, E. C. W. (2002). Psychosocial correlates of death anxiety among Chinese college students. Death Studies, 26(6), 491-499. https://doi.org/10.1080/074811802760139012

Tabachnick, B. G. \& Fidell, L. S. (2013). Using multivariate statistics (6a ed). Pearson Education.

Templer, D. I. (1970). The construction and validation of a Death Anxiety Scale. The Journal of General Psychology, 82(2), 165-177. https://doi.org/10.1080/00221309.1970.9920634

Tomás-Sábado, J. \& Gómez-Benito, J. (2002). Psychometric properties of the Spanish form of Templer's Death Anxiety Scale. Psychological Reports, 91(3_suppl), 1116-1120. https://doi.org/10.2466/pr0.2002.91.3f.1116

Tomás-Sábado, J. \& Gómez-Benito, J. (2003). Variables relacionadas con la ansiedad ante la muerte. Revista de Psicología General y Aplicada, 56(3), 257-279. https://dialnet.unirioja.es/servlet/articulo?codigo=818725

Tomás-Sábado, J. \& Gómez-Benito, J. (2004). Note on death anxiety in Spain and five Arab countries. Psychological Reports, 95, 12391240. https://doi.org/10.2466/pr0.95.3f.1239-1240

Tomás-Sábado, J. \& Gómez-Benito, J. (2005). Construction and validation of the Death Anxiety Inventory (DAI). European Journal of Psychological Assessment, 21(2), 108. https://doi.org/10.1027/1015-5759.21.2.108

Tomás-Sábado, J., Limonero, J. T. \& Gómez-Benito, J. (1-28 de febrero de 2007). Ansiedad ante la muerte y ansiedad general. ¿Dos constructos diferenciados? [Presentación de ponencia]. VIII Congreso Virtual de Psiquiatría (Interpsiquis 2007). Bibliopsiquis.

Tong, E., Deckert, A., Gani, N., Nissim, R., Rydall, A., Hales, S., Rodin, G. \& Lo, C. (2016). The meaning of self-reported death anxiety in advanced cancer. Palliative Medicine, 30(8), 772-779. https://doi.org/10.1177/0269216316628780

Fecha de recepción: Junio de 2018.

Fecha de aceptación: Junio de 2020. 